In the sexual response cycle, aging affects the stages of desire, arousal/excitement,plateau, orgasm, and resolution/refractory period both men and women.

Depressed mood as well as the use of antidepressant drugs, deterioration of cognitive function and difficulties to communicate can cause loss of intimacy and emotional closeness which then may result in sexual withdrawal and difficulties. Repetitive experiences of failure increase even more the couple distress.

There are also to consider medical conditions, medication side effects, loss of physical capacity, subjective loss of attractiveness, lack of a partner, institutionalization or living withtheir children, which can be obstacles to sexual activity in the elderly.

Diagnostic workup has to integrate not only general medical, gynecological, urological andpsychiatric factors, but also take a systemic perspective which deals with the interaction pattern of the partners. Treatment of sexual dysfunctions in later life combines biomedical interventions with psychotherapeutic and psychosocial strategies. An essential therapeutic contribution for allcouples is, however, basic counseling and psychoeducation. Explaining how aging has an impact and how other individuals and couples experience these changes is an important step in empowering the couple, clarifying and correcting the sociocultural myths andencouraging the couple to build their very own sexuality.

\title{
OnDemand Poster
}

\section{1 - Prediction of Mild Cognitive Impairment $(\mathrm{MCl})$ progression to Alzheimer Disease (AD) or Dementia with Lewy Bodies (DLB): Is this possible neuropsychologically? \\ Panagiota Voskou, PhD, MD, MSc,}

Objective: Aim of the present review study was to describe and compare the neurocognitive features of $\mathrm{MCl}$ which could predict its progression to DLB vs AD.

Background: Progression of $\mathrm{MCI}$ to $\mathrm{AD}$ or $\mathrm{DLB}$ is a relatively recent field of study with emphasis on the clinical or neuropsychological features of $\mathrm{MCl}$ which could potentially predict its progression to specific types of dementia.

Methods: A literature review in the Pubmed database has been made, after the year 2005, using the keywords: neuropsychological assessment; $\mathrm{MCl}$; $\mathrm{AD}$; DLB; progression to dementia. Seventeen relevant articles have been found.

Results: Data from most studies supports that, in $\mathrm{MCl}$, impairment in executive, attentional and visuospatial functions, as well as letter fluency and fluctuating concentration are mainly related to progression to DLB. In contrast, prominent episodic and recognition memory deficits are mostly found in $\mathrm{MCl}$ which progresses in $\mathrm{AD}$. Furthermore, non-amnestic $\mathrm{MCl}$ has been related most often to progression in $D L B$, whereas the amnestic type to $A D$, although memory loss may not necessarily predict the development of AD. Nevertheless, fewer studies suggest that $\mathrm{MCI}-\mathrm{DLB}$ is related to cognitive profile similar to that of $\mathrm{MCl}-\mathrm{AD}$, while cognitive scoring alone does not accurately predict MCI-DLB vs MCl-AD. Interestingly, quantitative electroencephalogram has been found to help in predicting the progression of $\mathrm{MCl}$ to DLB, while preservation of hippocampal volume is associated with increased risk of DLB vS AD, especially in non-amnestic $\mathrm{MCl}$. Moreover, specific patterns on neuroimaging $\mathrm{MCl}$ may predict progression to $A D$ in contrast to DLB. 
Conclusions: Predicting the progression of $\mathrm{MCl}$ to $A D$ or DLB based on neuropsychological profiles is challenging and useful for early therapeutic interventions. More studies are needed, since there are some conflicting findings and, at present, the combination of clinical symptoms with neurocognitive assessment and neuroimaging is the ideal method for the prediction of $\mathrm{MCl}$ progression to various types of dementia.

\section{2 - Diagnosis of dementia and post-diagnostic support - voice of people with dementia living in Poland}

Authors:

Maćkowiak, M., Ciułkowicz, M, Duda-Sikuła, M., Szcześniak, D., Rymaszewska, J. on behalf of the COGNISANCE Team

Background:

The existing guidelines for the process of dementia diagnosis and treatment does not necessarily translate into optimal care and support for people with dementia. Such situation can have significant consequences. Misunderstanding of the new life situation as well as the negative perception of the diagnostic process itself are intensified by the limited options of post-diagnostic support. Especially in Poland, where the national dementia strategy has still not been implemented, experiences of people undergoing diagnosis and seeking support should be heard. By giving voice to people with dementia we can value their personal experiences, which due to the existing stigma, may often be overlooked and neglected.

\section{Objective:}

The aim of the study is to examine factors (conditions, barriers, facilitators) to receiving a dementia diagnosis and post-diagnostic support from the perspective of people with dementia living in Poland. The research was conducted as part of the JPND project COGNISANCE.

\section{Methods:}

Ten people with dementia diagnosed within last 24 months were interviewed with the use of selected projective techniques (word associations and third person expressive technique). An inductive qualitative content analysis approach has been implemented to analyse the obtained data.

\section{Results:}

Analysis revealed diverse categories encompassing broad spectrum of conditions, barriers and facilitators to receive a diagnosis and post-diagnostic support. People with dementia were prone to feeling positive emotions despite negative initial reactions during the diagnosis. Positive adaptation to the dementia were highly dependent on the empathetic attitude of the doctor and supportive family. Main needs were related to maintaining the independence, staying active, healthy and being socially connected. Interpersonal factors ensured sense of security and stability crucial for the well-being.

\section{Conclusions:}

Presented results provide important insight into the current experiences of people with dementia and represent guidelines for care providers and policy makers in introducing the optimal solutions in dementia support. 